\title{
Irreversible Primary Visual Cortex Impairment in a Mouse Model of High-Risk Schizophrenia
}

\begin{abstract}
Xinying Chen ${ }^{1, *}$, Ce Chen ${ }^{2, *}$, Feng $\mathrm{ji}^{3, *}$, Yong $\mathrm{Xu}^{4}$, Wenqiang Wang ${ }^{5}$, Xiaodong Lin $^{2}$, Deguo Jiang ${ }^{2}$, Xueqin Song ${ }^{6}$, Xiangyang $\mathrm{Gao}^{7}$, Hongjun $\operatorname{Tian}^{8}$, Chuanjun Zhuo ${ }^{8}$, jingliang Zhang ${ }^{9}$

'Psychiatric-Neuroimaging-Genetics-Comorbidity Laboratory (PNGC_Lab), Tianjin Mental Health Centre, Mental Health Teaching Hospital of Tianjin Medical University, Tianjin Anding Hospital, Tianjin 300222, People's Republic of China; ${ }^{2}$ PsychiatricNeuroimaging-Genetics Laboratory, Wenzhou Seventh People's Hospital, Wenzhou, Zhejiang Province 325000, People's Republic of China; ${ }^{3}$ Department of Psychiatry, School of Mental Health, Jining Medical University, Jining 272I I9, Shandong Province, People's Republic of China; ${ }^{4}$ Department of Psychiatry, First Hospital/First Clinical Medical College of Shanxi Medical University, MDT Center for Cognitive Impairment and Sleep Disorders, First Hospital of Shanxi Medical University, Taiyuan 03000I, People's Republic of China; ${ }^{5}$ Co-Collaboration Laboratory of China and Canada, Xiamen Xianyue Hospital and University of Alberta, Xiamen 361000, People's Republic of China; ${ }^{6}$ The First Affiliated Hospital/ Zhengzhou University, Biological Psychiatry International Joint Laboratory of Henan/ Zhengzhou University, Henan Psychiatric Transformation Research Key Laboratory/ Zhengzhou University, Zhengzhou 450052, People's Republic of China; 'Health Management Institute, Center for Statistical Analysis of Medical Data, Medical Big Data Analysis Center, Chinese PLA General Hospital, Beijing 100191, People's Republic of China; ${ }^{8}$ Department of Neurology and Psychiatry Biological Imaging Laboratory (NPBI_Lab), Tianjin Fourth Center Hospital, Tianjin 200024, People's Republic of China; ${ }^{9}$ Department of Psychiatry, Wenzhou Kangning Hospital, Wenzhou, Zhejiang Province 325007, People's Republic of China

*These authors contributed equally to this work
\end{abstract}

Correspondence: Chuanjun Zhuo

Department of Neurology and Psychiatry

Biological Imaging Laboratory (NPBI_Lab),

Tianjin Fourth Center Hospital, Tianjin 200024,

People's Republic of China

Tel/Fax +86-22-24394542

Email chuanjunzhuotjmh@ieee.org

Jingliang Zhang

Department of Psychiatry, Wenzhou Kangning Hospital, Wenzhou, Zhejiang Province 325007,

People's Republic of China

Email zhangill2141029@sina.com

\author{
This article was published in the following Dove Press journal: \\ Neuropsychiatric Disease and Treatment
}

Purpose: Although visual deficits can be observed at any stage of schizophrenia, few studies have focused on visual cortex alterations in individuals at high risk of schizophrenia. This study aimed to investigate the pathological changes of the primary visual cortex in a prenatal mouse model of MK801-induced high-risk schizophrenia.

Methods: The high-risk schizophrenia model was generated by MK801 injection into pregnant mice. The male offspring without schizophrenia-like behaviors in early adulthood were defined as the high-risk mouse model of schizophrenia (HRMMS) and divided into two groups. One HRMMS group received the antipsychotic agent risperidone beginning at postnatal week 4 and another group did not receive any treatment. After treatment for 4 weeks, in vivo two-photon calcium imaging was performed to characterize the primary visual cortex activity. The novel object recognition test and the prepulse inhibition apparatus test were also implemented to assess the cognitive and behavioral performance, respectively. Results: Both groups of HRMMS mice, with or without antipsychotic treatment, had decreased neuronal calcium activity, demonstrating primary visual cortex impairment. More notably, antipsychotic treatment did not normalize the impaired neuronal activities in the primary visual cortex. Correspondingly, the treatment did not improve the cognitive or behavioral impairment.

Conclusion: Visual cortex impairment might be a prominent feature of individuals at high risk of schizophrenia that cannot be normalized by early treatment with antipsychotic medication, indicating the presence of independent regulatory pathways for visual perception disturbance in schizophrenia. Thus, visual system impairment in schizophrenic patients must be further studied.

Keywords: schizophrenia, animal model, primary visual cortex, antipsychotics

\section{Introduction}

Numerous studies have reported that more than half of patients with schizophrenia experience visual perception disturbances, such as visional hallucination, visional distortions, etc., ${ }^{1}$ which can be detected at any stage of schizophrenia. Moreover, such visual perception disturbances can be observed in individuals with a high risk of schizophrenia. ${ }^{2,3}$ The visual disturbances can further deteriorate other psychotic symptoms, including auditory impairment, cognitive deficits, memory dysfunction, and other symptoms of mental disorders. ${ }^{4-6}$ To date, the effect of antipsychotic medicines on the visual disturbances in patients with schizophrenia remains unclear. $^{7,8}$ Since visual disturbances often occur in high-risk individuals or in the early stage of schizophrenia, further understanding of its pathological features should help to facilitate the early prevention of schizophrenia and to avoid further 
disease deterioration. ${ }^{9,10}$ Thus, it is necessary to investigate the functional alterations of the visual cortex under schizophrenia conditions.

During the last decade, many studies have reported visual disturbances in patients with schizophrenia that are frequently accompanied with structural and functional alterations of the brain, based on brain imaging during visual perception tasks. For example, Silverstein et al have described that patients with schizophrenia presented increased neural activity in the fusiform area when performing the spatial frequency-degraded faces task. ${ }^{11,12}$ In addition, Nagel et al have demonstrated unique extra-retinal neuronal characteristics during smooth pursuit eye movements in patients with schizophrenia. ${ }^{13}$ Moreover, Lencer et al have reported reduced neuronal activity in the visual cortex and its association with smooth-pursuit deficits. ${ }^{14}$ Furthermore, Onitsuka et al have found a reduced gray matter volume of the occipital lobe in male patients with schizophrenia. ${ }^{15}$ Additionally, Calderone et al have shown disturbances of electrophysiological recording and blood oxygen signals during the visual contrast responses task in patients with schizophrenia. ${ }^{16,17}$ Schizophrenic patients also had impairment of dynamic coupling in the lateral occipital complex during visual perception, as reported by Harvey et al. ${ }^{18}$ In summary, these studies provide evidence that correlates visual disturbances with brain pathology in schizophrenic patients.

The aforementioned studies suggest the importance of visual perception disturbances in patients with schizophrenia as well as in individuals with a high risk of schizophrenia. They all recommended further exploration of the pathological features of the visual pathway (especially the primary visual cortex alterations) by multiple techniques in individuals who are at high risk of schizophrenia. Simultaneously, some studies have proposed to analyze the effects of antipsychotic agents on the visual perception disturbance and the corresponding brain activity alterations in order to explore strategies for early disease prevention and management. ${ }^{9}$

Considering the urgent need to understand the visual pathway alterations in individuals at high risk of schizophrenia, we designed a pilot animal study to investigate the functional changes in the primary visual cortex. In this novel mouse model of high-risk schizophrenia, we aimed to answer the following questions: 1) Compared to healthy controls, do offspring mice at high risk of schizophrenia have primary visual cortex functional impairment?; and 2) Does early treatment with the antipsychotic agent risperidone protect the primary visual cortex?

\section{Materials and Methods}

\section{Animals}

Pregnant C57BL/6 mice were housed in an animal facility with food and water available ad libitum. To create the mouse model of pregnant mice with schizophrenia, a single intraperitoneal injection of MK801, also known as dizocilpine, a noncompetitive N-methyl-D-aspartic acid receptor antagonist that induces schizophrenia-like symptoms, was administered at gestation day $7-19(0.1 \mathrm{mg} / \mathrm{kg}$ by subcutaneous injection). After the offspring mice were born, the surviving male offspring were selected as study subjects. When the offspring mice were 4 weeks old, their behavior was assessed by the sucrose preference test and the prepulse inhibition (PPI) apparatus test. ${ }^{19}$ The novel object recognition (NOR) test was performed according to previously published methods. ${ }^{20}$ For the PPI test, a $120-\mathrm{dB}$ (40 ms) startle was applied after a $20-\mathrm{ms}$ prepulse at 75 $\mathrm{dB}$, with a time interval of $100 \mathrm{~ms}$. The background noise was controlled at $65 \mathrm{~dB}$. The inter-trial time was set at 30 s. Generally, three sessions were used, and the scores were averaged. The offspring mice were divided into two categories according to their performance in the PPI test: mice without schizophrenic behaviors and mice with schizophrenic behaviors. Each category of mice was further divided into two groups: one group was administered with the antipsychotic agent risperidone for prevention (from the age of 4 weeks old to 8 weeks old), while the other group did not receive any treatment. Only 21 mice survived until the age of 8 weeks, including $9(9 / 10$, surviving/total) mice without schizophrenic behaviors and without antipsychotic agent prevention, 7 (7/10, surviving/total) mice without schizophrenic behaviors but with antipsychotic agent prevention, 3 (3/10, surviving/total) mice with schizophrenic behaviors but without treatment, and 2 (2/10, surviving/total) mice with schizophrenic behaviors and treatment. All experimental procedures on animals were approved by the Animal Ethics Committee of Jining Medical University, The First Hospital of Shanxi Medical University, Tianjin Mental Health Centre (IRB number: JSTEBSRA-001) in accordance with the guidelines of Institutional Animal Care and Use Committee (IACUC).

\section{In vivo Calcium Recordings and Analysis}

The neuronal activity in the visual cortex was determined according to previously published methods, ${ }^{21}$ with slight modifications. In brief, anaesthetized mice were fixed, and a chronic cranial window was created. Then, $200 \mathrm{~nL}$ of the 
adeno-associated virus $2 / 9$-syn-GCaMP6s virus $\left(2 \times 10^{13}\right.$ genome copies $/ \mathrm{mL}$; University of Pennsylvania Vector Core Facility) was injected bilaterally into the primary visual cortex using the following coordinates: $-3.1 \mathrm{~mm}$ from the bregma, $\pm 2.5 \mathrm{~mm}$ to the midline. The imaging window was covered by a circular coverslip, and the skull was sealed using dental cement. A customized steel bar was embedded into the skull for fixation of the mouse head during the imaging session.

For two-photon in vivo imaging, previously reported approaches were followed. ${ }^{22}$ A two-photon microscope (LSM780; Zeiss, Germany) was used with a $16 \times, 0.8$ numerical aperture water-immersion objectively. Using an excitation wavelength of $950 \mathrm{~nm}$, time-series images were recorded at $1.96 \mathrm{~Hz}$ for $150-\mathrm{s}$ periods. Captured images were analyzed using ImageJ software (National Institutes of Health, Bethesda, MD, USA). Regions of interest were selected manually in ImageJ with the FIJI plug-in package, ${ }^{23}$ followed by the detection and normalization of the calcium transients. In general, for each recording time-series, the basal values $\left(\mathrm{F}_{0}\right)$ were averaged based on the first 15 -sec values under resting state. Fluorescent values in each series were then normalized and transformed into relative changes $\left(\Delta \mathrm{F}=\left(\mathrm{F}-\mathrm{F}_{0}\right) / \mathrm{F}_{0}\right)$.

\section{Statistical Analysis}

All experimental data are presented as the mean \pm standard error of mean, unless otherwise specified. ${ }^{21}$ The twosample Student's $t$-test or nonparametric KolmogorovSmirnov test was used to compare means between two groups. ${ }^{21}$ For multi-group comparisons, one-way analysis of variance was performed, followed by Tukey's post hoc comparison. ${ }^{21}$ GraphPad Prism 7.0 was used for statistical analyses and data plotting $(P<0.05) .{ }^{21}$

\section{Results}

\section{Dysregulation of Visual Cortical Activities in Mouse Models at High Risk of Schizophrenia}

Utilizing in vivo two-photon imaging, the calcium activities in the layer $2 / 3$ pyramidal neurons in the primary visual cortex were recorded in awake and head-fixed mice, which had been transfected with the genetically encoded calcium indicator GCaMP6s using an adenoassociated virus vector. The time-elapsed recordings of neuronal activity demonstrated that all groups at high risk of schizophrenia (with or without early antipsychotic agent intervention) had significantly lower calcium activities compared to the healthy control mice (One-way ANOVA, F $(4,35)=13.63, P<0.001$; Tukey's multiple comparison test, $P<0.001$ between control and HRMMS, and between control and HRMMS+Risperidone; Figure $1 \mathrm{~A}$ and $\mathrm{B})$. Moreover, no significant difference was observed between mice with and without early antipsychotic agent intervention (Figure 1A and B).

Regretfully, in this pilot study, we did not successfully acquire the in vivo two-photon imaging and staining imaging from all the model mice. We only successfully acquired the imaging of seven mice (total nine mice) without schizophrenic behaviors and without early antipsychotic agent's prevention, five mice (total seven mice) without schizophrenic behaviors but with antipsychotic agent prevention, two mice (total three mice) with schizophrenic behaviors but without early antipsychotic agent's prevention, and none of mice (total two mice) with schizophrenic behaviors and with early antipsychotic agent's prevention.

\section{Effectiveness of Risperidone in the Early Prevention of Disease Phenotypes}

Both PPI and NOR tests demonstrated that the early usage of the antipsychotic agent risperidone failed to prevent visual cortical neural activity in mice with a high risk of schizophrenia (PPI: One-way ANOVA, F $(4,35)=14.22$, $P<0.001$; NOR: One-way ANOVA, F $(4,30)=9.83$, $P<0.001$; Tukey's multiple comparison test, $P<0.001$ between control and HRMMS, and between control and HRMMS+Risperidone; Figure 1C and D). Moreover, we also tested if there are side effects of Risperidone on naïve mice by those two behavioral paradigms. Results showed that the early application of Risperidone did not cause significant change of either PPI or NOR functions.

\section{Discussion}

To the best of our knowledge, the present pilot study provides the first piece of evidence of visual impairments including sensory integration in the visual cortex using in vivo two-photon imaging on a mouse model of highrisk schizophrenia. In addition, we also investigated the effect of the early administration of the antipsychotic agent risperidone on normalization of the functional deficits. Two phenomena generated from this pilot study are worth further investigations.

First, we found impairment of neural activity in the primary visual cortex in mice with a high risk of 
A
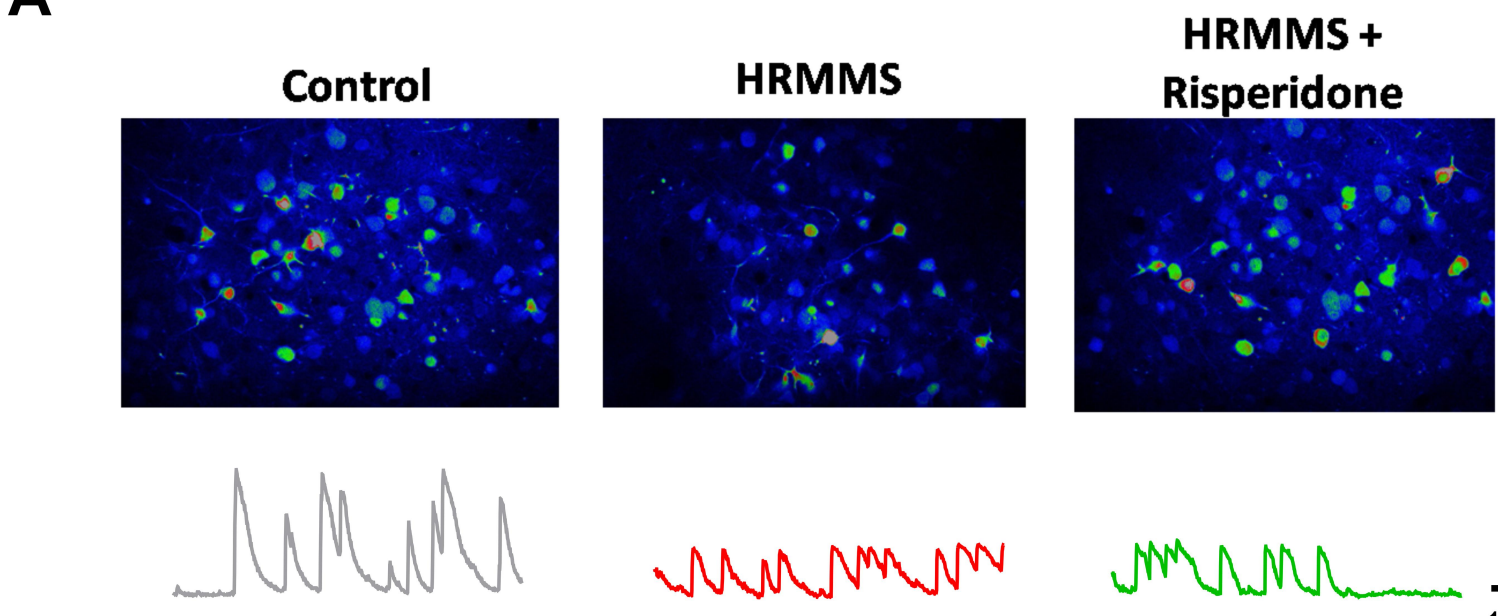

B

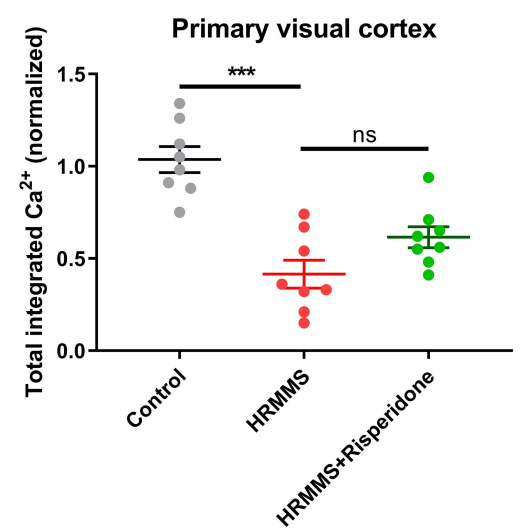

C

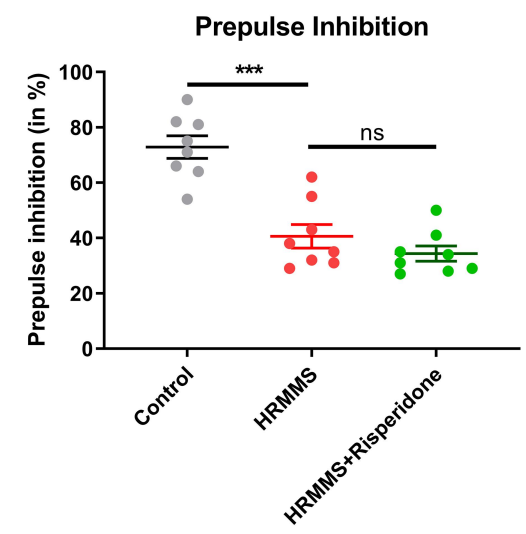

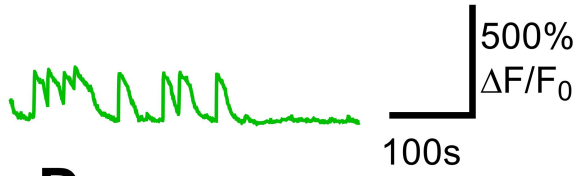

D

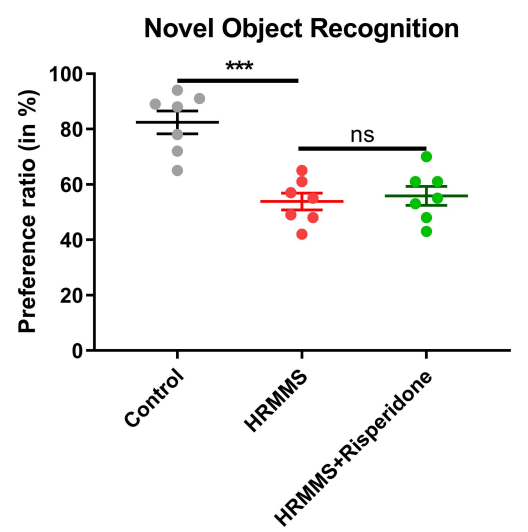

Figure I The differences in visual cortex function by in vivo two-photon calcium imaging (A and $\mathbf{B})$ as well as behavioral (C and $\mathbf{D})$ performance among the healthy control group, the high-risk mouse model of schizophrenia (HRMMS) group, and the HRMMS + risperidone group.

developing schizophrenia. This finding provides evidence that brain function impairments occur even before psychotic episodes take place. Thus, it is likely that such deficits in the visual cortex might occur in individuals with a high risk of schizophrenia. In fact, many functional magnetic resonance imaging studies in humans have reported the existence of structural and functional impairments in the visual cortex in individuals with a high risk of schizophrenia. ${ }^{11-13,16}$ Our pilot study agreed with these previous studies, supporting the hypothesis that visual impairment might be one early marker of schizophrenia that can aid in disease diagnosis.

Second, and perhaps most importantly, early intervention using the antipsychotic agent risperidone did not restore normal function of the primary visual cortex or improve the cognitive and behavioral performance. Some magnetic resonance imaging studies have reported that early intervention using antipsychotic agents can protect against brain impairment in individuals at a high risk of developing schizophrenia, ${ }^{24,25}$ but we did not observe any improvement of neural activity by two-photon imaging in the animal model. In contrast, one previous study ${ }^{9}$ has reported that antipsychotic agent administration to patients with schizophrenia reduced the effect on the primary visual cortex. We suggest that such discrepancy and ineffectiveness of drug intervention may exist in the time point of treatment initiation. At week 4, most of the brain circuits have been functionally matured, and further drug treatment may not dramatically change the neural circuit deformation and dysfunction. In future, the early intervention may help to improve psychiatric disorders caused by prenatal stress.

There are several limitations of this pilot study that must be mentioned. First, since the survival rate of the mice in this model was relatively low, survivor bias may exist, thus affecting the interpretation of the results. Second, we only observed the visual cortex impairment 
but not retina alterations in model mice. Hence, our finding cannot fully represent the visual system impairment in model mice. In future studies, in vivo two-photon imaging and optical coherence tomography will be performed to investigate the mice at high risk of schizophrenia systematically to further explore the full visual pathway (from visual organization $[\mathrm{eye}]^{26,27}$ to the primary and high visual cortex) as well as the structural and functional alterations so that prevention and intervention strategies for schizophrenia can be developed.

In conclusion, despite certain limitations, this pilot study in animals provides the first piece of evidence showing impairments of the visual cortex in a model of schizophrenia. More importantly, this pilot study showed that the impairments in the visual cortex could not be reversed and, in some cases, were even further deteriorated by treatment with an antipsychotic agent. In summary, our findings indicate that visual cortex impairment might be a marker of schizophrenia that cannot be normalized by early treatment with antipsychotic medication. Thus, there is an urgent need to address the issue of visual system impairments in patients with schizophrenia.

\section{Funding}

This work was supported by grants from the National Natural Science Foundation of China (81871052 to C.Z., 81801679 and 81571319 to Y.X.), the Key Projects of the Natural Science Foundation of Tianjin, China (17JCZDJC35700 to C.Z.), the Tianjin Health Bureau Foundation (2014KR02 to C.Z.), the National Key Research and Development Program of China (2016YFC1307004 to Y.X.), the Shanxi Science and Technology Innovation Training Team's Multidisciplinary Team for Cognitive Impairment (201705D131027 to Y. X.), the Zhejiang Public Welfare Fund Project (LGF18H090002 to D.J), the key project of the Wenzhou Science and Technology Bureau (ZS2017011 to X.L), Tianjin 131 Scholar Project to C.Z, and Tianjin Anding Hospital of 300000 Scholar Award Project to C.Z.

\section{Disclosure}

The authors declare that they have no conflicts of interest.

\section{References}

1. Waters F, Collerton D, Ffytche DH, et al. Visual hallucinations in the psychosis spectrum and comparative information from neurodegenerative disorders and eye disease. Schizophr Bull. 2014;40(Suppl 4): S233-S245. doi:10.1093/schbul/sbu036
2. Mittal VA, Gupta T, Keane BP, Silverstein SM. Visual context processing dysfunctions in youth at high risk for psychosis: resistance to the Ebbinghaus illusion and its symptom and social and role functioning correlates. $J$ Abnorm Psychol. 2015;124:953-960. doi:10.1037/abn0000082

3. Hebert M, Gagne AM, Paradis ME, et al. Retinal response to light in young nonaffected offspring at high genetic risk of neuropsychiatric brain disorders. Biol Psychiatry. 2010;67:270-274. doi:10.1016/j. biopsych.2009.08.016

4. Grano N, Salmijarvi L, Karjalainen M, Kallionpaa S, Roine M, Taylor P. Early signs of worry: psychosis risk symptom visual distortions are independently associated with suicidal ideation. Psychiatry Res. 2015;225:263-267. doi:10.1016/j.psychres.2014.12.031

5. Clark A. Whatever next? Predictive brains, situated agents, and the future of cognitive science. Behav Brain Sci. 2013;36:181-204. doi:10.1017/S0140525X12000477

6. Green MF, Hellemann G, Horan WP, Lee J, Wynn JK. From perception to functional outcome in schizophrenia: modeling the role of ability and motivation. Arch Gen Psychiatry. 2012;69:1216-1224. doi:10.1001/archgenpsychiatry.2012.652

7. Guidotti A, Grayson DR. A neurochemical basis for an epigenetic vision of psychiatric disorders (1994-2009). Pharmacol Res. 2011;64:344-349. doi:10.1016/j.phrs.2011.05.026

8. Fernandes TMP, Silverstein SM, Butler PD, et al. Color vision impairments in schizophrenia and the role of antipsychotic medication type. Schizophr Res. 2019;204:162-170. doi:10.1016/j.schres.20 18.09.002

9. Silverstein SM. Visual perception disturbances in schizophrenia: a unified model. Nebr Symp Motiv. 2016;63:77-132.

10. Silverstein SM, Rosen R. Schizophrenia and the eye. Schizophr Res Cogn. 2015;2:46-55. doi:10.1016/j.scog.2015.03.004

11. Silverstein SM, All SD, Kasi R, et al. Increased fusiform area activation in schizophrenia during processing of spatial frequency-degraded faces, as revealed by fMRI. Psychol Med. 2010;40:1159-1169. doi:10.1017/S0033291709991735

12. Silverstein SM, Berten S, Essex B, Kovacs I, Susmaras T, Little DM. An fMRI examination of visual integration in schizophrenia. $J$ Integr Neurosci. 2009;8:175-202. doi:10.1142/S0219635209002113

13. Nagel M, Sprenger A, Nitschke M, et al. Different extraretinal neuronal mechanisms of smooth pursuit eye movements in schizophrenia: an fMRI study. Neuroimage. 2007;34:300-309. doi:10.1016/ j.neuroimage.2006.08.025

14. Lencer R, Nagel M, Sprenger A, Heide W, Binkofski F. Reduced neuronal activity in the V5 complex underlies smooth-pursuit deficit in schizophrenia: evidence from an fMRI study. Neuroimage. 2005;24:1256-1259. doi:10.1016/j.neuroimage.2004.11.013

15. Onitsuka T, McCarley RW, Kuroki N, et al. Occipital lobe gray matter volume in male patients with chronic schizophrenia: a quantitative MRI study. Schizophr Res. 2007;92:197-206. doi:10.1016/j.schres.2007.01.027

16. Calderone DJ, Martinez A, Zemon V, et al. Comparison of psychophysical, electrophysiological, and fMRI assessment of visual contrast responses in patients with schizophrenia. Neuroimage. 2013;67:153-162. doi:10.1016/j.neuroimage.2012.11.019

17. Foxe JJ, Doniger GM, Javitt DC. Early visual processing deficits in schizophrenia: impaired P1 generation revealed by high-density electrical mapping. Neuroreport. 2001;12:3815-3820. doi:10.1097/0000 1756-200112040-00043

18. Harvey PO, Lee J, Cohen MS, et al. Altered dynamic coupling of lateral occipital complex during visual perception in schizophrenia. Neuroimage. 2011;55:1219-1226. doi:10.1016/j.neuroimage.2010. 12.045

19. Hass K, Bak N, Szycik GR, Glenthoj BY, Oranje B. Deficient prepulse inhibition of the startle reflex in schizophrenia using a cross-modal paradigm. Biol Psychol. 2017;128:112-116. doi:10.10 16/j.biopsycho.2017.07.016 
20. Zieba J, Morris MJ, Weickert CS, Karl T. Behavioural effects of high fat diet in adult Nrg1 type III transgenic mice. Behav Brain Res. 2020;377:112217. doi:10.1016/j.bbr.2019.112217

21. Chen K, Zheng Y, Wei JA, et al. Exercise training improves motor skill learning via selective activation of mTOR. Sci Adv. 2019;5: eaaw1888. doi:10.1126/sciadv.aaw 1888

22. Forster D, Dal Maschio M, Laurell E, Baier H. An optogenetic toolbox for unbiased discovery of functionally connected cells in neural circuits. Nat Commun. 2017;8:116. doi:10.1038/s41467-017-00160-z

23. Hutchinson EB, Stefanovic B, Koretsky AP, Silva AC. Spatial flow-volume dissociation of the cerebral microcirculatory response to mild hypercapnia. Neuroimage. 2006;32:520-530. doi:10.1016/j. neuroimage.2006.03.033

24. Iwashiro N, Koike S, Satomura Y, et al. Association between impaired brain activity and volume at the sub-region of Broca's area in ultra-high risk and first-episode schizophrenia: a multimodal neuroimaging study. Schizophr Res. 2016;172:9-15. doi:10.1016/j.schres.2016.02.005
25. Kasparek T, Prikryl R, Schwarz D, et al. Gray matter morphology and the level of functioning in one-year follow-up of first-episode schizophrenia patients. Prog Neuropsychopharmacol Biol Psychiatry. 2009;33:1438-1446. doi:10.1016/j.pnpbp.2009.07.025

26. Chu EM, Kolappan M, Barnes TR, Joyce EM, Ron MA. A window into the brain: an in vivo study of the retina in schizophrenia using optical coherence tomography. Psychiatry Res. 2012;203:89-94. doi:10.1016/j.pscychresns.2011.08.011

27. Schonfeldt-Lecuona C, Kregel T, Schmidt A, et al. From imaging the brain to imaging the retina: optical coherence tomography (OCT) in schizophrenia. Schizophr Bull. 2016;42:9-14. doi:10.1093/schbul/ sbv073

\section{Publish your work in this journal}

Neuropsychiatric Disease and Treatment is an international, peerreviewed journal of clinical therapeutics and pharmacology focusing on concise rapid reporting of clinical or pre-clinical studies on a range of neuropsychiatric and neurological disorders. This journal is indexed on PubMed Central, the 'PsycINFO' database and CAS, and is the official journal of The International Neuropsychiatric Association (INA). The manuscript management system is completely online and includes a very quick and fair peer-review system, which is all easy to use. Visit http://www.dovepress.com/testimonials.php to read real quotes from published authors. 